\title{
Child development, growth and microbiota: follow-up of a randomized education trial in Uganda
}

Prudence Atukunda ${ }^{1}$, Grace K M Muhoozi ${ }^{1,2}$, Tim J van den Broek ${ }^{3}$, Remco Kort ${ }^{3,4}$, Lien M Diep ${ }^{5}$, Archileo N Kaaya $^{6}$, Per O Iversen ${ }^{1,7,8, *}$, Ane C Westerberg,"*

${ }^{1}$ Department of Nutrition, Institute of Basic Medical Sciences, University of Oslo, Oslo, Norway

${ }^{2}$ Department of Human Nutrition and Home Economics, Kyambogo University, Kampala, Uganda

${ }^{3}$ Netherlands Organization for Applied Scientific Research (TNO), Microbiology and Systems Biology, Zeist, the Netherlands

${ }^{4}$ Department of Molecular Cell Biology, VU University Amsterdam, the Netherlands

${ }^{5}$ Oslo Centre for Biostatistics and Epidemiology, Oslo University Hospital, Oslo, Norway

${ }^{6}$ School of Food Technology, Nutrition and Bioengineering, Makerere University, Kampala, Uganda

${ }^{7}$ Department of Haematology, Oslo University Hospital, Oslo, Norway

${ }^{8}$ Division of Human Nutrition, Stellenbosch University, Tygerberg, South Africa

${ }^{9}$ Institute of Health Sciences, Kristiania University College, Oslo, Norway

* Equal authorship

\section{Correspondence to:}

Per Ole Iversen

Department of Nutrition

P.O. Box 1046 Blindern

0317 Oslo

Norway

p.o.iversen@medisin.uio.no
Background Undernutrition impairs child development outcomes and growth. In this follow-up study of an open cluster-randomized intervention trial we examined the effects of an education package delivered to mothers in rural Uganda on their children's development, growth and gut microbiota at 36 months of age.

Methods The parental trial included 511 mother-child pairs recruited when the children were 6-8 months. In that trial, a nutrition, stimulation and hygiene education was delivered to mothers in the intervention group while the control group received routine health care. A follow-up sample of 155 pairs (intervention $n=77$, control $n=78$ ) were re-enrolled when the children were 24 months. Developmental outcomes were assessed with the Bayley Scales of Infant and Toddler Development (BSID-III) composite scores for cognitive (primary endpoint), language and motor development. Development outcomes were also evaluated using the Ages and Stages Questionnaire (ASQ) and the Mullen Scales of Early Learning (MSEL). Other outcomes included growth and gut microbiota composition.

Results The demographic characteristics were not different $(P>0.05)$ between the intervention and control groups and similar to those of the parental study. The intervention group had higher BSID-III scores than controls, with mean difference 10.13 (95\% confidence interval (CI): 3.31-17.05, $P=0.002) ; 7.59$ (1.62-13.66, $P=0.01)$; 9.00 (2.92-15.40, $P=0.005)$, for cognitive, language and motor composite scores, respectively. An improvement in the intervention compared to the control group was obtained for both the ASQ and the MSEL scores. The mean difference in height-for-age z-score was higher in the intervention compared to the control group: $0.50(0.25-0.75$, $P=0.0001$ ). Gut microbiota composition did not differ significantly between the two study groups.

Conclusions The maternal education intervention had positive effects on child development and growth at three years, but did not alter gut microbiota composition. This intervention may be applicable in other low-resource settings.

Trial registration ClinicalTrials.gov registration number NCT02098031.

Undernutrition among children in developing countries is a major, global health challenge causing more than one-third of under-five deaths [1]. About 200 million children below five years worldwide who are undernourished do not meet their development potential [2]. In addition to stunting, poor cognitive stimulation, and adverse environmental conditions, low maternal education is recognized as risk factors for impaired child development [3]. In line with this, undernourished children 
are at risk of impaired structural development of the central nervous system (CNS) and extra- neuronal tissue [4]. Adequate childhood nutrition thus promotes healthy growth and development outcomes [5-7].

The underlying causes of chronic undernutrition are complex. In addition to inadequate food quantity and quality of food, a combination of poor sanitation and hygiene resulting in sustained exposure to enteric pathogens plays an important role [8]. Pregnancy and the first two years of life are important periods for interventions to improve child growth and cognitive development, and can be considered both a window of vulnerability as well as a window of opportunity [9]. The development of the gut microbiota is mostly accomplished within the first three years of life. Interventions directed towards an appropriate maturation of the gut microbiota and its associated metabolic potential, may support healthy growth and cognitive development [10]. In line with this, there are new insights into how the CNS and cognitive development may be influenced by the gut microbiota through the so-called "gut-brain axis" [11]. Moreover, a number of studies report systematic differences in the composition between rural Africa and urban Europe, indicating that the gut microbiota is tailored to local diet, specific nutritional requirements of the host and hygiene practices [12-14]. However, a possible role of gut microbiota in undernutrition and child development has not been adequately addressed. Inadequate caregiving skills and stimulation can also adversely impact development and growth of small children, in particular in low-resource settings. Integration of nutrition and stimulation in a Pakistani trial highlighted a potential for child development and linear growth benefits [5].

In 2013 we initiated a cluster-randomized controlled trial to examine the effect on growth and development of a 6-month intervention comprised of nutrition, stimulation and hygiene education among impoverished mothers of children aged 6-8 months in rural districts of Uganda [15]. The intervention consisted of educating mothers aimed at (i) increasing dietary diversity to improve nutrient intake as well as continued breastfeeding, (ii) improving hygiene and sanitation practices, and (iii) enhancing stimulation based on a social-cognitive learning theory to improve development. Whereas this intervention did not alter child growth at the age of 20-24 months, cognitive, language and motor development improved markedly [15]. In order to examine if these findings were sustainable over time, we decided to perform a follow-up study. Notably, a long term follow-up of such a nutrition education intervention has previously not been done in a resource-constrained setting as Uganda. We now examined development, growth and gut microbiota composition among a subsample of these children at the age of 36 months.

\section{METHODS}

\section{Study design and approvals}

This is a follow-up study of a two-armed, open cluster-randomized education intervention regarding nutrition, stimulation and hygiene among impoverished mothers of children aged 6-8 months in the Kisoro and Kabale districts of South-Western Uganda. Details of the intervention have recently been published [15]. All mothers gave written or thumb-printed, informed consent to participate and could decline an interview or assessment at any time. The study was approved by The AIDS Support Organisation Research Ethics Committee (No. TASOREC/06/15-UG-REC-009) and by the Uganda National Council for Science and Technology (No. UNCST HS 1809) as well as by the Norwegian Regional Committee for Medical and Health Research Ethics (No. 2013/1833). The trial was registered with clinicaltrials. gov (NCT02098031). We report the data according to the CONSORT guidelines.

\section{Randomisation of the parental and follow-up participants}

For the parental trial we used proportionate sampling, 10 sub-counties (ie, clusters) were obtained ( 6 out of 19 in Kabale and 4 out of 14 in Kisoro) to participate in the study. We used a three-stage procedure to identify households for the study. First, by simple random sampling, three sub-counties in Kabale were allocated to the intervention group and the other three to the control group. Similarly, two sub-counties were allocated to the intervention and the other two to the control group in Kisoro district. Second, all the villages in each participating sub-county (intervention or control) were listed alphabetically and assigned numbers in an ascending order. By use of computer-generated random numbers, villages to whose assigned number matched with the random numbers were selected. The intervention villages did not share common geographical boundaries with control villages to minimize contamination of the intervention contents between the two study groups. Third, by complete enumeration, all consenting households with children aged 6-8 months within a participating village were recruited to the study. If a household had 
more than one eligible child, the youngest was selected, and in the case of twins, we randomly selected one for evaluation. We finally enrolled 511 mother-child pairs in the parental study and they were randomised to the intervention $(n=263)$ or the control $(n=248)$ group. The intervention group received the nutrition, hygiene and stimulation education in addition to routine health care while the control group received only routine health care.

The child had to be 20-24 months during the period of January-May 2015 to be included in the current follow-up study since age dynamic gut microbial shifts occur at this age resulting in an adult-like, stable composition [16], and developmental milestones at this age may predict IQ at 5-6 years when children are about to start school [17]. Based on a sample size calculation we then randomly selected participants from each of the two study groups ( $\mathrm{n}=77$ from the intervention group and $\mathrm{n}=78$ from the control group). Data was collected when the children were 20-24 months and at 36 months. The data collection teams in the follow-up study were masked to group allocation and never had any interaction with the study team that delivered the education intervention in the parental trial.

\section{Contents of the education intervention in the parental trial}

The intervention was conducted by the study team at three group meetings over a period of 6 months to 26 groups of mothers (6-10 mothers per group), and was detailed recently [15]. Briefly, it was delivered by a trained education team and included two behavior change techniques: providing information and prompt practice (ie, demonstrations of preparing food and stimulation of the children). The nutrition education curriculum was based on the 10 guiding principles of complementary feeding [18]. Recipes were formulated and cooking demonstrated using locally available foods with emphasis on protein. Moreover, the need to take ill children to hospital for medical attention and to increase the feeding frequency during and after illness was emphasized. Hand-washing before feeding as well as use of clean utensils during food preparation and feeding was part of the hygiene intervention. A novel aspect of this intervention was the focus on oral hygiene, and with distribution of tooth brushes to all household members and demonstration of their use. The education team highlighted the importance of play to improve cognitive, language and motor development. The stimulation intervention was based on social-cognitive learning theory [19]. In addition to the three group meetings, the women met at monthly intervals to practice what they had learnt and ensuring compliance to the intervention [15].

\section{Assessments of outcomes}

The child development assessments were performed by three bachelor degree holders in psychology whereas two graduates of laboratory technology collected stool samples. Two bachelor degree holders in nutrition collected the anthropometric data. These three data collection teams participated in training sessions to ensure uniform and standardized procedures. Assessments were administered in the local language and conducted in hired, secluded rooms in the villages without interruptions to minimize distractions. To promote reliability, the child development assessments were administered first, followed by anthropometric measurements, stool sampling and then interviews with the mothers.

The Bayley Scales of Infant and Toddler Development-III (BSID-III), the Ages and Stages Questionnaire (ASQ) and the Mullen Scales of Early Learning (MSEL; Supplementary information) were used [15]. The BSID-III scale is known to be the most comprehensive child development measure for children up to 3.5 years and has been adapted and used in similar settings [20]. The ASQ is a parent/caregiver completed screening scale with excellent psychometric properties which capture and establish a wide range of adaptive behaviors, and previously used in this setting [21]. Both tools were used because we did not include the social-emotional scale of BSID-III. The BSID-III and the ASQ were administered at 20-24 and at 36 months. MSEL was introduced at 36 months to assess early intellectual development and readiness for school, and it has been validated for use in rural Uganda [22]. Inter-observation agreement between the child assessment team was good indicated by an intra-class correlation coefficient (ICC) of 0.75 $(P=0.0001)$ for BSI-III, $0.79(P=0.0001)$ for ASQ and $0.77(P<0.001)$ for MSEL.

Weight, height, and head circumference (HC) were measured as recommended by WHO [21], with a Seca-scale model 881 (Hamburg, Germany) to the nearest $0.1 \mathrm{~kg}$. Height was measured (to the nearest $0.1 \mathrm{~cm}$ ) with a Seca board (SO114530). HC was measured with a non-stretchable tape (Seca, S0145630 PAC-50). Anthropometric data were converted to z-scores, height-for-age (HAZ), weightfor-age (WAZ), weight-for-height (WHZ), and head circumference (HCZ), using the Anthro (version 3.2.2) software, a nutritional assessment tool based on WHO standards. A $z$-score $<-2$ SD from the me- 
dian of the WHO reference standards indicated stunting for HAZ, underweight for WAZ and wasting for WHZ, respectively [23].

We collected stool samples using sterile cotton swabs (COPAN Diagnostics Inc, Murrieta, CA) and frozen at $-20^{\circ} \mathrm{C}$ within 24 hours of collection. The samples were then air-dried and shipped to the Netherlands for further processing and analyses (Supplementary information). These storage conditions have a very limited effect on the microbial composition [24]. All 16S rRNA amplicon paired end reads $(\mathrm{n}=560)$ of the gut microbiota samples sequenced in this study can be accessed at Sequence Read Archive (SRA) SUB4476421.

\section{Statistical analyses}

The primary outcome in the current follow-up study was cognitive development assessed with the BSIDIII at 36 months. Previous intervention studies in similar low-resource-settings report a mean difference of about $0.5 \mathrm{SD}$ in child development score between intervention and control groups [5,25]. To detect a difference between the two study groups in the BSID-III cognitive composite score at 36 months of $0.5 \mathrm{SD}$ (corresponding to 7.5 points) with a power of 0.8 and $\alpha$ of 0.05 , 63 children per group was required. To account for an intra-cluster correlation of 0.01 and dropouts, the mean number of children per sub-county was 15, thus a total of 155 children were included [15,26]. Among these 155 we randomly selected the 77 children from the intervention group and the 78 children from the control group at 20-24 months. Child development outcomes and growth were analyzed using Stata/SE (StataCorp. 2015, Stata Statistical Software: Release 14. College Station, Stockholm, Sweden) and SPSS version 22.0 (IBM SPSS Statistics, IBM Corp., Armonk, NY). Significance level was set at $P<0.05$. We used a mixed effect linear regression to compare the intervention with the control group and estimated ICC. Differences between the two study groups are given as mean (SD or 95\% CI).

All statistical analysis of gut microbiota on the 16S rRNA amplicon sequencing data was performed using $\mathrm{R}$ version 3.3.2 (R Core Team, 2016) [27]. The 16S rRNA amplicon sequencing data was rescaled and transformed using Wisconsin double and square root transformations. The PERMANOVA procedures, Shannon and 1-Simpson's diversity indices were performed as implemented in the 'vegan' package [28]. Whereas increasing values for the Shannon diversity index indicate more diversity, the opposite is true for the 1-Simpson's index. All PERMANOVA analyses were performed using the Bray-Curtis distance measure. All phyla and genera were included in the statistical analysis.

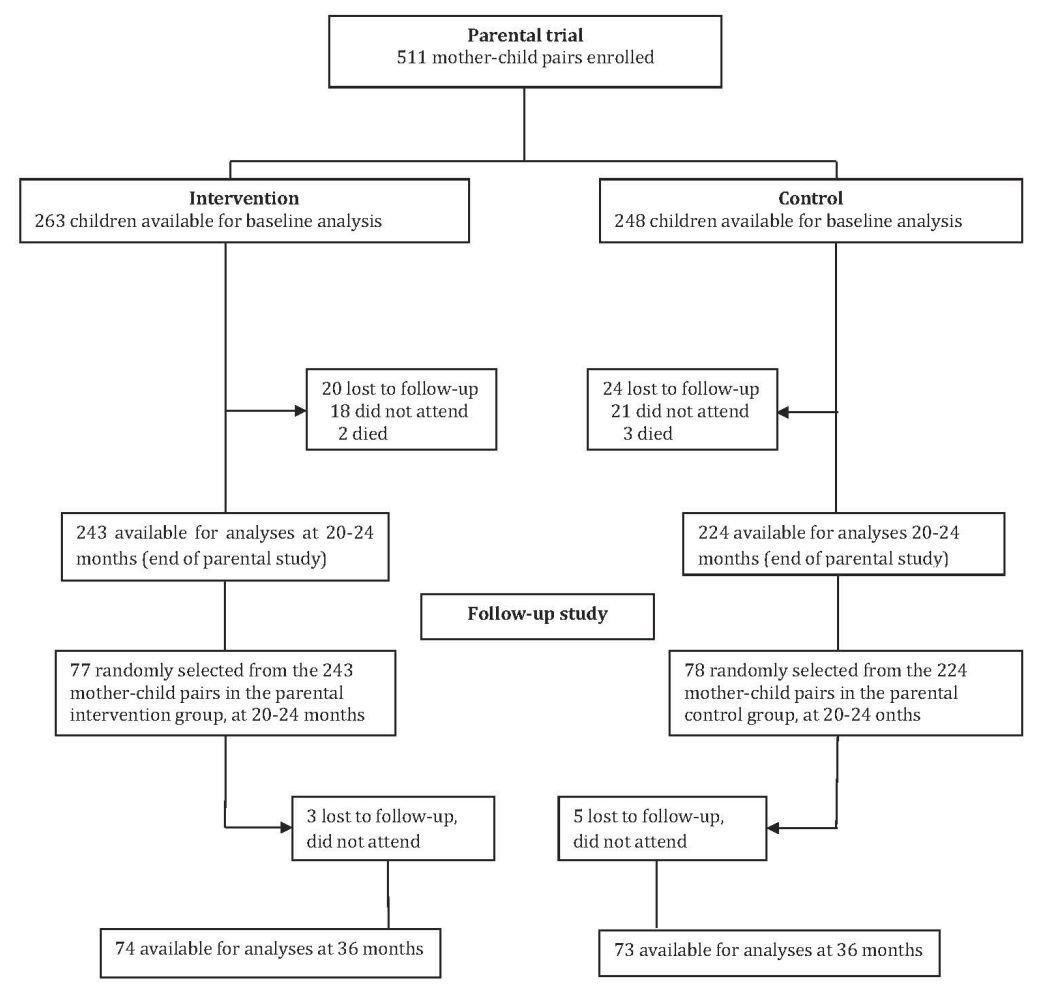

Figure 1. Profile of the parental trial and the follow-up study.

\section{RESULTS Study participants}

One hundred and fifty-five mother-child pairs were included at 20-24 months (Figure 1). By 36 months, eight of them were lost to follow-up (three in the intervention group and five in the control group). There were no significant differences in the characteristics between the parental cohort (data obtained at baseline) and the follow-up cohort (data obtained at 20-24 months; Table 1), thus no adjustments for baseline differences were made in subsequent analyses.

\section{Development outcomes}

Overall, the intervention significantly improved all child development outcomes (ie, cognitive, language and motor composite scores) based on the BSID-III at 36 months (Table 2). The Cohen's d effect sizes at 36 months were medium (cognitive 0.57 , language 0.56 and motor 0.50 ). The effect of the intervention on the ASQ mean scores for communication, gross motor, problem solving, and personal social development, was sig- 
Table 1. Study population characteristics for the parental trial at baseline and at start of the follow-up study*

\begin{tabular}{|c|c|c|c|c|c|}
\hline \multirow[t]{2}{*}{ Сharacteristics } & \multicolumn{2}{|c|}{ Pabental trial (data obtained at baseline) } & \multicolumn{2}{|c|}{ FoLLOW-UP study (DATA OBtalmed at 20-24 моNтhS) } & \multirow[t]{2}{*}{ P-value } \\
\hline & Intervention $(n=263)$ & Control $(n=248)$ & Intervention $(\mathrm{n}=77)$ & Control $(n=78)$ & \\
\hline \multicolumn{6}{|l|}{ Children ( $n, \%)$ : } \\
\hline Males & $139(52.9)$ & $123(49.6)$ & $44(57.1)$ & $41(52.6)$ & 0.75 \\
\hline Females & $124(47.1)$ & $125(50.4)$ & $33(42.9)$ & $37(47.4)$ & 0.40 \\
\hline Age at inclusion (months) & $7.4(0.8)$ & $7.3(0.9)$ & $21.4(1.0)$ & $21.2(1.0)$ & 0.24 \\
\hline Stunting $\dagger$ & $55(20.9)$ & $70(28.0)$ & $32(18.1)$ & $46(36.6)$ & 0.06 \\
\hline Underweight $\dagger$ & $25(9.5)$ & $36(14.5)$ & $6(8.3)$ & $8(11.3)$ & 0.37 \\
\hline Wasting $\dagger$ & $12(4.6)$ & $12(4.8)$ & $3(4.2)$ & $2(2.8)$ & 0.50 \\
\hline \multicolumn{6}{|l|}{ BSID-III composite score: } \\
\hline Cognitive & $114.9(21.3)$ & $99.3(17.1)$ & $116.1(15.6)$ & $105.9(15.9)$ & 0.89 \\
\hline Language & $98.3(14.3)$ & $88.4(9.1)$ & $106.5(14.8)$ & $98.9(12.8)$ & 0.50 \\
\hline Motor & $113.7(18.9)$ & $99.1(14.3)$ & $122.3(18.7)$ & $113.3(19.9)$ & 0.49 \\
\hline \multicolumn{6}{|l|}{ ASQ scores: } \\
\hline Communication & $40.8(14.5)$ & $33.8(15.3)$ & $51.4(9.9)$ & $48.1(11.4)$ & 0.36 \\
\hline Gross motor & $52.8(10.3)$ & $46.9(13.8)$ & $55.6(7.0)$ & $53.8(9.7)$ & 0.51 \\
\hline Fine motor & $44.6(9.9)$ & $40.4(11.5)$ & $47.9(10.8)$ & $42.5(13.9)$ & 0.23 \\
\hline Problem solving & $49.5(11.7)$ & $40.6(13.1)$ & $44.0(12.3)$ & $40.1(12.7)$ & 0.24 \\
\hline Personal-social & $41.0(11.3)$ & $36.6(11.1)$ & $48.7(10.8)$ & $45.8(9.9)$ & 0.35 \\
\hline \multicolumn{6}{|l|}{ Illness at study time $(n, \%)$ : } \\
\hline Yes & $94(35.7)$ & $71(28.6)$ & $47(61.0)$ & $40(51.3)$ & 0.21 \\
\hline No & $169(64.3)$ & $177(71.4)$ & $30(39.0)$ & $38(48.7)$ & 0.38 \\
\hline \multicolumn{6}{|l|}{ Maternal data: } \\
\hline Maternal education (years) & $4.9(2.8)$ & $4.9(2.8)$ & $5.5(2.5)$ & $5.0(2.6)$ & 0.20 \\
\hline Maternal age (years) & $26.1(5.8)$ & $26.8(6.3)$ & $26.2(6.1)$ & $27.4(6.4)$ & 0.27 \\
\hline Number of children per mother & $3.4(2.2)$ & $3.3(2.2)$ & $3.4(2.2)$ & $3.3(2.2)$ & 0.25 \\
\hline \multicolumn{6}{|l|}{ Household data: } \\
\hline Household head age (years) & $31.3(7.7)$ & $32.6(19.4)$ & $30.2(7.3)$ & $33.1(10.9)$ & 0.06 \\
\hline Household head education (years) & $6.4(3.1)$ & $5.9(3.1)$ & $6.6(3.3)$ & $6.5(3.4)$ & 0.29 \\
\hline Household size (n) & $5.5(2.1)$ & $5.5(2.1)$ & $5.7(2.2)$ & $5.8(2.2)$ & 0.76 \\
\hline Household poverty score & $47.8(11.7)$ & $47.6(11.4)$ & $49.0(11.6)$ & $46.3(12.3)$ & 0.18 \\
\hline Sanitation composite score & $7.2(1.9)$ & $7.3(1.9)$ & $7.0(1.8)$ & $7.1(1.9)$ & 0.83 \\
\hline
\end{tabular}

ASQ - Ages and Stages Questionnaire, BSID - Bayley's Scales of Infant and Toddler Development

*Values are means (SD) unless otherwise stated.

$\dagger z$-score values are <-2SD of the median of the reference population.

Table 2. Composite scores derived from the Bayley Scales of Infant and Toddler Development-III scales*

\begin{tabular}{|c|c|c|c|c|c|}
\hline Age of chILD (MONths) & Intervention (n = 73-77)† & Control $(n=74-78) \dagger$ & BetweEN GROUP DIFFERENGE & \multirow{2}{*}{ P-valuE§ } & \multirow{2}{*}{ ICE } \\
\hline \multicolumn{4}{|c|}{ Cognitive composite scores: } & & \\
\hline $20-24$ & $117.84(20.86)$ & $101.58(19.14)$ & $16.26(9.57$ to 23.04$)$ & 0.0001 & 0.05 \\
\hline 36 & $116.07(15.55)$ & $105.94(15.99)$ & $10.13(3.31$ to 17.05$)$ & 0.002 & \\
\hline \multicolumn{6}{|c|}{ Language composite scores: } \\
\hline $20-24$ & $100.31(12.91)$ & $89.00(9.32)$ & $11.31(5.43$ to 17.28$)$ & 0.0001 & 0.06 \\
\hline 36 & $106.54(14.79)$ & $98.95(12.77)$ & 7.59 (1.62 to 13.66$)$ & 0.010 & \\
\hline \multicolumn{6}{|c|}{ Motor composite scores: } \\
\hline $20-24$ & $113.79(16.06)$ & $100.04(15.47)$ & $13.75(7.80$ to 20.01$)$ & 0.0001 & 0.01 \\
\hline 36 & $122.32(18.74)$ & $113.32(19.89)$ & $9.00(2.92$ to 15.40$)$ & 0.005 & \\
\hline
\end{tabular}

ICC - intra-class correlation coefficient

*Values are means (standard deviation) unless otherwise stated.

$\uparrow$ The variation in $\mathrm{n}$ was due to missing data because some children did not complete all the tests.

¥Mean differences (95\% CI) of Bayley Scales of Infant and Toddler Development-III composite scores.

$\S P$-value is for the difference between the two study groups adjusted for clusters.

nificantly higher in the intervention group compared with the controls at 24 months (Table 3). At 36 months, the ASQ fine motor scores were significantly higher in the intervention group compared with the controls. The Cohen's d effect sizes at 36 months ranged from small to medium for the ASQ scores (gross motor 0.16, personal social development 0.25, problem solving 0.29, fine motor 0.49 and communication 0.68). Also, the MSEL fine motor, language (receptive and expressive), cognitive and early 
Table 3. Mean scores from the Ages and Stages Questionnaire*

\begin{tabular}{|c|c|c|c|c|c|}
\hline Age of ChILD (MONThs) & Intervention (n= 71-74)† & Control $(n=70-73) \dagger$ & BetweEN GROUP DIFFERENCE & $P_{\text {-valueS }}$ & ICC \\
\hline \multicolumn{6}{|c|}{ Communication scores: } \\
\hline $20-24$ & $41.37(14.04)$ & $31.58(18.45)$ & 9.79 (3.90 to 15.76$)$ & 0.001 & 0.06 \\
\hline 36 & $51.41(9.96)$ & $48.11(11.40)$ & $3.30(-2.68$ to 9.33$)$ & 0.28 & \\
\hline \multicolumn{6}{|c|}{ Gross motor scores: } \\
\hline $20-24$ & $53.46(10.76)$ & $46.47(15.79)$ & 6.99 (2.47 to 11.60$)$ & 0.003 & 0.00 \\
\hline 36 & $55.58(7.04)$ & $53.80(9.72)$ & $1.78(-2.80$ to 6.47$)$ & 0.44 & \\
\hline \multicolumn{6}{|l|}{ Fine motor scores: } \\
\hline $20-24$ & $45.73(9.93)$ & $42.04(12.58)$ & $3.69(-0.27$ to 8.01$)$ & 0.067 & 0.07 \\
\hline 36 & $47.93(10.80)$ & 42.52 (13.94) & 5.41 (1.36 to 9.81$)$ & 0.010 & \\
\hline \multicolumn{6}{|c|}{ Problem solving scores: } \\
\hline $20-24$ & $50.35(10.19)$ & $38.94(14.24)$ & $11.41(7.24$ to 15.57$)$ & 0.0001 & 0.02 \\
\hline 36 & $44.02(12.25)$ & $40.06(12.69)$ & $3.96(-0.31$ to 8.21$)$ & 0.069 & \\
\hline \multicolumn{6}{|c|}{ Personal-social development scores: } \\
\hline $20-24$ & $43.24(10.41)$ & $36.81(10.05)$ & $6.43(1.99$ to 10.85$)$ & 0.0001 & 0.06 \\
\hline 36 & $48.74(10.83)$ & $45.75(9.95)$ & $2.99(-1.54$ to 7.49$)$ & 0.10 & \\
\hline
\end{tabular}

ICC - intra-class correlation coefficient

*Values are means (standard deviation) unless otherwise stated. ICC-intra-class correlation coefficient.

$\uparrow$ The variation in $\mathrm{n}$ was due to missing data because some children did not complete all the tests.

\$Mean differences (95\% CI) of Ages and Stages Questionnaire scores

$\S P$-value is for the difference between the two study groups adjusted for clusters.

learning composite standard scores were significantly higher in the intervention compared to the controls at 36 months (Table 4). In contrast, the MSEL visual reception scores were not different between the two study groups. The corresponding mean Cohen's d effect sizes were: $0.23,0.44,0.34,0.42,0.42$, and 0.36 for MSEL visual reception, fine motor, receptive language, expressive language, cognitive total score and early learning score, respectively.

\section{Growth outcomes}

The mean HAZ declined in both study groups during the study period, indicating linear growth faltering (Table 5). However, this decline was significantly less at 36 months in the intervention compared with the control group. There were no significant differences in the other mean anthropometric measures (ie, WAZ, WHZ, and HCZ) at 36 months. The Cohen's d effect sizes at 36 months were 1.01, 0.16, -0.46, and 0.30 for HAZ, WAZ, WHZ, and HCZ, respectively.

\section{Gut microbiota composition}

The intervention did not lead to any significant changes in the gut microbiota diversity compared with the control group at the phylum level (Figure 2). Neither did we observe any significant differences between the two study groups in the Shannon diversity index at the two time points (Figure 3). However, as expected the Shannon diversity index increased significantly in both study groups from 20-24 to 36 months, indicating increased gut microbiota diversity, while there was no significant change in the overall genera distribution from 20-24 to 36 months. In line with this, there was no change in the variable 1-Simpson index between the two study groups at the two time points (Figure 3), and this variable increased from 20-24 to 36 months, again indicating increased gut microbiota diversity. In support of these findings, the

Table 4. Mullen Scales of Early Learning scores obtained in the two study groups at 36 months*

\begin{tabular}{|c|c|c|c|c|}
\hline & InteRvention (n = 74) & Control $(n=73)$ & BetweEN GROUP DIFFERENCE† & P-value \\
\hline Visual reception & $53.31(13.63)$ & $50.33(12.44)$ & $2.98(-7.24$ to 1.27$)$ & 0.17 \\
\hline Fine motor & $62.84(15.55)$ & $56.18(14.91)$ & 6.66 (1.69 to 11.83$)$ & 0.009 \\
\hline Receptive language & $58.72(10.33)$ & $55.10(11.26)$ & $3.62(0.10$ to 7.14$)$ & 0.044 \\
\hline Expressive language & $60.59(10.33)$ & $56.25(10.51)$ & $4.34(0.95$ to 7.74$)$ & 0.012 \\
\hline Cognitive total score & $235.46(42.27)$ & $217.85(41.35)$ & 17.61 (3.98 to 31.24) & 0.012 \\
\hline Early learning score & $75.64(29.17)$ & $64.77(31.67)$ & 10.87 (1.81 to 14.87$)$ & 0.013 \\
\hline
\end{tabular}

*Values are means (standard deviation) unless otherwise stated.

$†$ Mean differences (95\% confidence interval) of Mullen Scales of Early Learning scores.

$\ddagger P$-value is for the difference between the two study groups adjusted for clusters. 
Table 5. Child growth during the study period*

\begin{tabular}{|c|c|c|c|c|c|}
\hline AgE Of CHILD (MONTHS) & Intervention ( $\mathrm{n}=74-77) \dagger$ & Control $(n=73-78) \dagger$ & BetweEN GROUP DIFFERENCE‡ & P-value $§$ & ICC \\
\hline \multicolumn{6}{|c|}{ Height-for-age z-scores: } \\
\hline $20-24$ & $-1.96(1.14)$ & $-2.07(1.20)$ & $0.11(-0.14$ to 0.35$)$ & 0.41 & 0.34 \\
\hline 36 & $-2.15(1.01)$ & $-2.65(0.88)$ & $0.50(0.25$ to 0.75$)$ & 0.0001 & \\
\hline \multicolumn{6}{|c|}{ Weight-for-age z-scores: } \\
\hline $20-24$ & $-0.76(0.88)$ & $-0.85(0.88)$ & $0.09(-0.37$ to 0.55$)$ & 0.70 & 0.10 \\
\hline 36 & $-0.98(0.89)$ & $-1.18(0.69)$ & $0.20(-0.27$ to 0.66$)$ & 0.40 & \\
\hline \multicolumn{6}{|c|}{ Weight-for-height z-scores: } \\
\hline $20-24$ & $0.26(0.94)$ & $0.45(0.77)$ & $-0.19(-0.52$ to 0.16 & 0.31 & 0.04 \\
\hline 36 & $0.44(0.91)$ & $0.84(0.74)$ & $0.40(-0.75$ to 0.05 & -0.054 & \\
\hline \multicolumn{6}{|c|}{ Head circumference z-scores: } \\
\hline $20-24$ & $0.30(0.93)$ & $0.61(1.05)$ & $-0.25(-0.64$ to 0.04$)$ & 0.079 & 0.00 \\
\hline 36 & $-0.34(0.90)$ & $0.05(1.01)$ & $-0.39(-0.72$ to 0.34$)$ & 0.055 & \\
\hline
\end{tabular}

*Values are means (standard deviation) unless otherwise stated. ICC-intra-class correlation coefficient.

The variation in $\mathrm{n}$ is due to missing data.

¥Mean differences (95\% confidence interval).

$\S P$-value is for the difference between the two study groups adjusted for clusters.

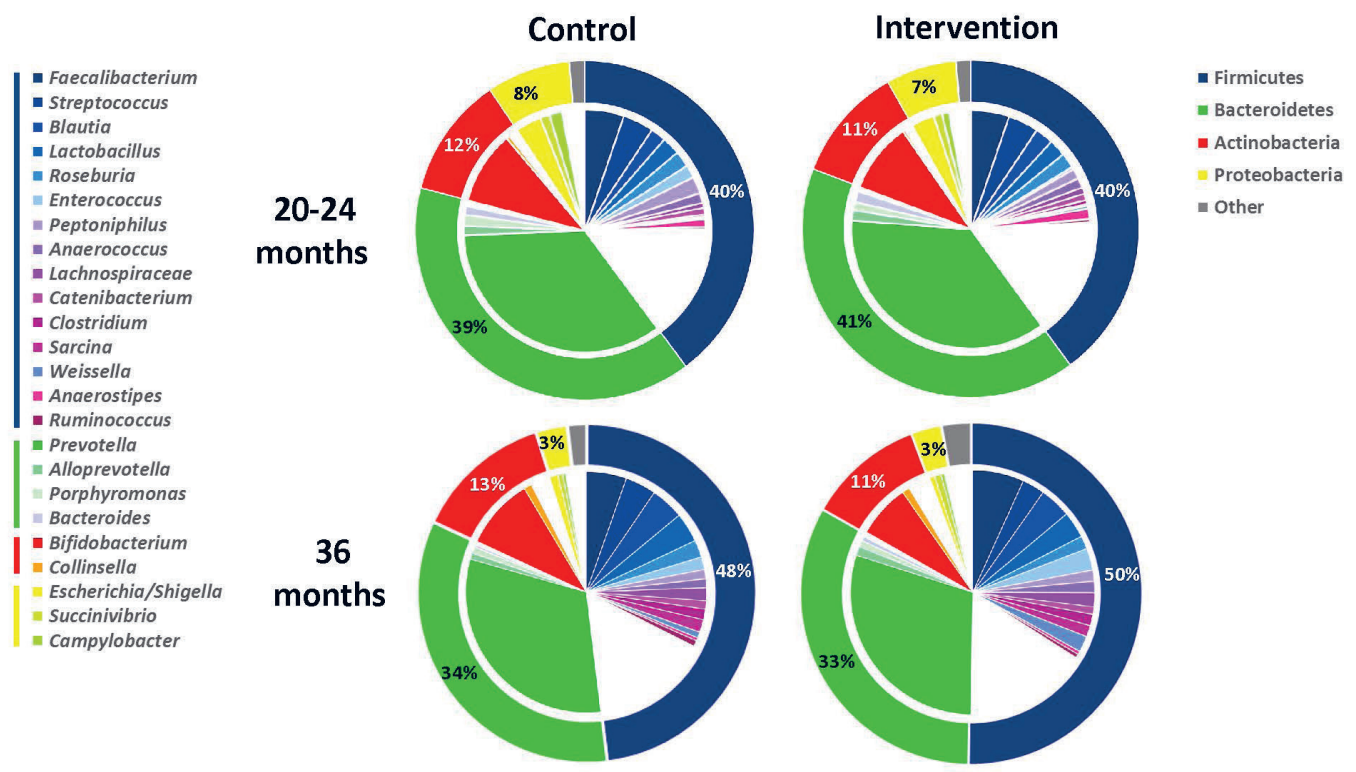

Figure 2. Fecal microbiota compositions based on normalized $16 \mathrm{~S}$ rRNA amplicon sequencing reads from the control (left pie charts) and intervention (right pie charts) group at 20-24 (upper pie charts) and at 36 (lower pie charts) months. The outer donuts represent the four predominant phyla (legend: right upper corner) and the inner pie charts the most abundant genera within each of these phyla (legend: left). Charts indicate the average relative abundance of phyla and genera in the fecal microbiota of the children with a cut-off value of $0.7 \%$.

PERMANOVA analysis revealed that there was a significant change in the composition of the gut microbiota from 20-24 to 36 months, both at the genus $(P=0.001)$ and at the phylum $(P=0.001)$ level, but that there was no significant effect $(P=1)$ of the intervention on the overall gut microbiota composition.

\section{DISCUSSION}

This is probably the first randomized education intervention trial incorporating gut microbiota analysis in rural Sub-Saharan Africa. In the parental trial the 6-month education intervention led to significant improvements in development outcomes when the children reached 20-24 months, without affecting growth [15]. We now show a sustained improvement in the development outcomes even at 36 months and with the use of three independent tools. The intervention also reduced linear growth faltering until 36 months, but had no effect on gut microbiota composition. 

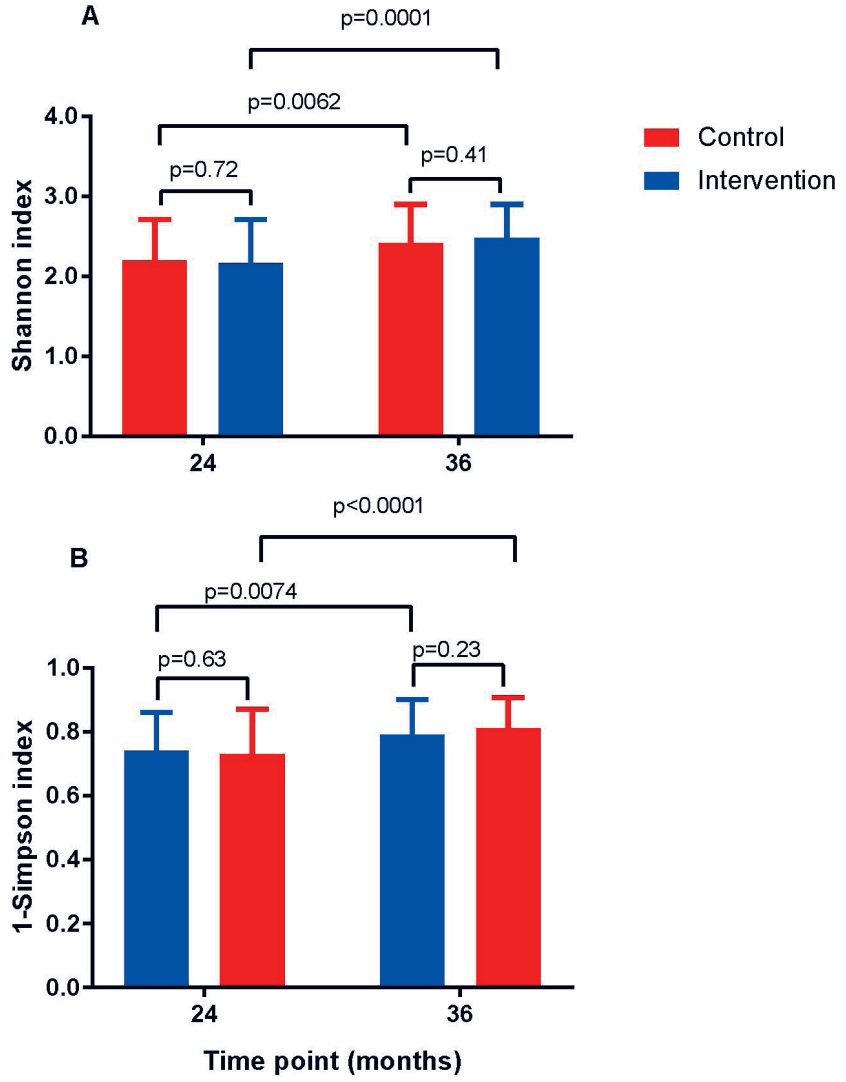

Figure 3. Shannons diversity index (A) and 1-Simpsons index (B) for gut microbiota diversity obtained from the control (open symbol) and intervention (closed symbol) group among the children at age 20-24 and 36 months. Values are mean \pm standard deviation.
Our effect sizes on child development outcomes were comparable/higher than those obtained in previous studies that included nutrition supplementation and child stimulation intervention $[5,29,30]$. Studies from low income countries using the BSID-III to assess development found that children who received both nutrition and responsive stimulation reported higher cognitive, language and motor skills compared to those who only received either nutrition or stimulation [25,31]. Notably, most of these studies provided micronutrient supplementation and play materials whereas we educated the mothers without supporting them with either food or toys. Mother-child play interaction models promote children's engagement in several activities that enhance development [32]. Moreover, our findings are in accordance with a previous Ugandan study which reported slightly higher cognitive scores three months after stimulation and nutrition education [20].

The baseline stunting levels observed in the two study groups compare favorably with those of previous surveys in Uganda [33]. Corroborating the anthropometric results obtained when the children were 20-24 months [15], most growth indicators were not significantly different between the two study groups at 36 months. The only exception was a smaller reduction in linear growth faltering in the intervention compared with the control group. This could imply that the education intervention may have a protective effect against linear growth faltering over time.

Emerging data suggest links between gut microbiota composition and stunting as well as cognition in childhood [34,35], possibly mediated through cross-talk between microbiota-derived signaling molecules and host tissues [36]. As improved diet and hygienic practices may promote a healthy gut microbiota [37], interventions to enhance nutrition may indirectly impact positively on child growth and development outcomes $[38,39]$. Previous studies on nutrition and gut microbiota are mostly based on animal models or clinical trials with specific nutrients, pre- or probiotics to modify microbiota diversity [39]. In the present trial we emphasized education of the mothers about preparing nutritious foods, ensuring hygienic meal preparations and maintaining good oral health among their children. Despite acceptable adherence to this intervention [15], we could not detect any significant effects on gut microbiota composition after 20-24 or after 36 months.

Our baseline data on maternal and household characteristics were in line with previous reported data from Uganda [40-42]. Our education intervention consisted of a combined strategy to improve nutrient intakes, hygiene/sanitary practices and stimulation through increased knowledge and empowerment of the mothers. Although it is not possible to exactly specify which component(s) led to the improvement in child development outcomes, the unchanged child diet diversity observed among the households in the intervention group at 20-24 months [15] as well as the unaltered gut microbiota, suggest that the improvements were predominantly resulting from enhanced stimulation and hygiene practices. A systematic review of combined nutrition and stimulation interventions reported that child development was consistently improved through stimulation while growth and nutritional status were usually improved by nutrition [43]. Although this review found little evidence for combined benefits of both nutrition and child stimulation interventions on child development, our findings indicate that having a combination of nutrition, hygiene and child stimulation education may have a potential benefit on child development outcomes.

\section{Strengths and limitations}

In this study we adopted a multidisciplinary approach combining aspects of nutrition, hygiene, psychology, microbiology and validated research instruments. Of note, the children were followed for several years. Despite that only about one-third of the mother-child pairs of the parental trial could be re-enrolled for this follow-up study, the latter cohort was well balanced with the baseline characteristics of the parental 
cohort. A limitation of our study was lack of baseline data of gut microbiota composition, and we have no information about body composition, dietary intakes or relevant biomarkers among the children or if the mothers in the intervention group continued to stimulate their children in the period between end of intervention and when the children reached the age of three years. ASQ is a maternal report and could possibly be biased. Furthermore, we do not report on maternal mental health which may impact on development and growth of small children, in particular in low-resource settings [44].

\section{CONCLUSIONS}

This nutrition, hygiene and stimulation education intervention among mothers of 6-8 months old children had a positive effect on child development and growth until 36 months. We found no significant effects of the intervention on gut microbiota composition. The positive effects from this intervention would call for further research of such an intervention before consideration of scale-up and implementation in other low-income rural settings.

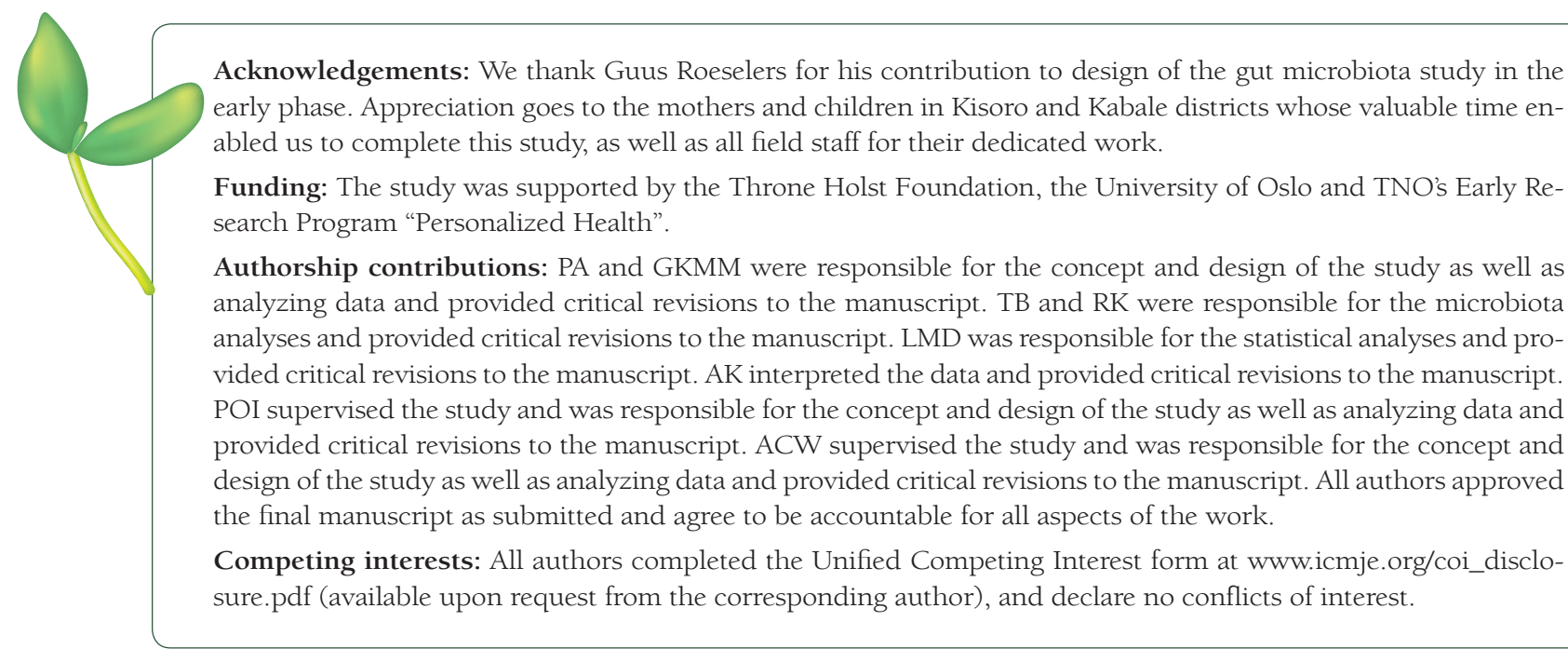

1 Black RE, Allen LH, Bhutta ZA, Caulfield LE, de Onis M, Ezzati M, et al. Maternal and child undernutrition: global and regional exposures and health consequences. Lancet. 2008;371:243-60. Medline:18207566 doi:10.1016/S01406736(07)61690-0

2 Countdown to 2030 Collaboration. Countdown to 2030: tracking progress towards universal coverage for reproductive, maternal, newborn, and child health. Lancet. 2018;391:1538-48. Medline:29395268 doi:10.1016/S01406736(18)30104-1

$3 \mathrm{Lu}$ C, Black MM, Richter LM. Risk of poor development in young children in low-income and middle-income countries: an estimation and analysis at the global, regional, and country level. Lancet Glob Health. 2016;4:e916-22. Medline:27717632 doi:10.1016/S2214-109X(16)30266-2

4 Prado EL, Dewey KG. Nutrition and brain development in early life. Nutr Rev. 2014;72:267-84. Medline:24684384 doi:10.1111/nure.12102

5 Yousafzai AK, Rasheed MA, Rizvi A, Armstrong R, Bhutta ZA. Effect of integrated responsive stimulation and nutrition interventions in the Lady Health Worker programme in Pakistan on child development, growth, and health outcomes: a cluster-randomised factorial effectiveness trial. Lancet. 2014;384:1282-93. Medline:24947106 doi:10.1016/S01406736(14)60455-4

6 Aboud FE, Yousafzai AK. Global health and development in early childhood. Annu Rev Psychol. 2015;66:433-57. Medline:25196276 doi:10.1146/annurev-psych-010814-015128

7 Larson LM, Yousafzai AK. A meta-analysis of nutrition interventions on mental development of children under-two in low- and middle-income countries. Matern Child Nutr. 2017;13. Epub 2015 Nov 26. Medline:26607403 doi:10.1111/ mcn.12229

8 Kane AV, Dinh DM, Ward HD. Childhood malnutrition and the intestinal microbiome. Pediatr Res. 2015;77:256-62. Medline:25356748 doi:10.1038/pr.2014.179

9 Krebs NF, Lozoff B, Georgieff MK. Neurodevelopment: The impact of nutrition and inflammation during infancy in low-resource settings. Pediatrics. 2017;139 Suppl 1:S50-8. Medline:28562248 doi:10.1542/peds.2016-2828G 
10 Gordon JI, Dewey KG, Mills DA, Medzhitov RM. The human gut microbiota and undernutrition. Sci Transl Med. 2012;4:137ps12. Medline:22674549 doi:10.1126/scitranslmed.3004347

11 Sherwin E, Sandhu KV, Dinan TG, Cryan JF. May the force be with you: The light and dark sides of the microbiota-gut-brain axis in neuropsychiatry. CNS Drugs. 2016;30:1019-41. Medline:27417321 doi:10.1007/s40263-016-0370-3

12 De Filippo C, Cavalieri D, Di Paola M, Ramazzotti M, Poullet JB, Massart S, et al. Impact of diet in shaping gut microbiota revealed by a comparative study in children from Europe and rural Africa. Proc Natl Acad Sci U S A. 2010;107:146916. Medline:20679230 doi:10.1073/pnas.1005963107

13 Grześkowiak Ł, Collado MC, Mangani C, Maleta K, Laitinen K, Ashorn P, et al. Distinct gut microbiota in southeastern African and northern European infants. J Pediatr Gastroenterol Nutr. 2012;54:812-6. Medline:22228076 doi:10.1097/ MPG.0b013e318249039c

14 Cheung YB, Xu Y, Mangani C, Fan YM, Dewey KG, Salminen SJ, et al. Gut microbiota in Malawian infants in a nutritional supplementation trial. Trop Med Int Health. 2016;21:283-90. Medline:26644222 doi:10.1111/tmi.12650

15 Muhoozi GKM, Atukunda P, Diep LM, Mwadime R, Kaaya AN, Skaare AB, et al. Nutrition, hygiene, and stimulation education to improve growth, cognitive, language, and motor development among infants in Uganda: A cluster-randomized trial. Matern Child Nutr. 2018;14:e12527. Medline:28925580 doi:10.1111/mcn.12527

16 Yatsunenko T, Rey FE, Manary MJ, Trehan I, Dominguez-Bello MG, Contreras M. etal. Human gut microbiome viewed across age and geography. Nature. 2012;486:222-7. Medline:22699611 doi:10.1038/nature11053

17 Peyre H, Charkaluk ML, Forhan A, Heude B, Ramus F. Do developmental milestones at 4, 8, 12 and 24 months predict IQ at 5-6 years old? Results of the EDEN mother-child cohort. Eur J Paediatr Neurol. 2017;21:272-9. Medline:27889381 doi:10.1016/j.ejpn.2016.11.001

18 PAHO/WHO. Guiding Priciples for Complementary Feeding of the Breastfed Child. Washington D.C, Division of Health Promotion and Protection; World Health Organisation; 2003.

19 Bandura A. Social cognitive theory: an agentic perspective. Annu Rev Psychol. 2001;52:1-26. Medline:11148297 doi:10.1146/annurev.psych.52.1.1

20 Singla DR, Kumbakumba E, Aboud FE. Effects of a parenting intervention to address maternal psychological wellbeing and child development and growth in rural Uganda: a community-based, cluster randomised trial. Lancet Glob Health. 2015;3:e458-e469. Medline:26144389 doi:10.1016/S2214-109X(15)00099-6

21 Hornman J, Kerstjens JM, de Winter AF, Bos AF, Reijneveld SA. Validity and internal consistency of the Ages and Stages Questionnaire 60-month version and the effect of three scoring methods. Early Hum Dev. 2013;89:1011-5. Medline:24041814 doi:10.1016/j.earlhumdev.2013.08.016

22 Boivin MJ, Sikorskii A, Familiar-Lopez I, Ruiseñor-Escudero H, Muhindo M, Kapisi J, et al. Malaria illness mediated by anaemia lessens cognitive development in younger Ugandan children. Malar J. 2016;15:210. Medline:27076184 doi:10.1186/s12936-016-1266-x

23 Child Growth Standards WHO. Geneva, Switzerland:World Health Organization; 2006.

24 Lauber CL, Zhou N, Gordon JI, Knight R, Fierer N. Effect of storage conditions on the assessment of bacterial community structure in soil and human-associated samples. FEMS Microbiol Lett. 2010;307:80-6. Medline:20412303 doi:10.1111/ j.1574-6968.2010.01965.x

25 Gardner JM, Powell CA, Baker-Henningham H, Walker SP, Cole TJ, Grantham-McGregor SM. Zinc supplementation and psychosocial stimulation: effects on the development of undernourished Jamaican children. Am J Clin Nutr. 2005;82:399405. Medline:16087985 doi:10.1093/ajcn/82.2.399

26 Campbell MJ, Donner A, Klar N. Developments in cluster randomized trials and statistics in medicine. Stat Med. 2007;26:2-19. Medline:17136746 doi:10.1002/sim.2731

27 Team RC. A language and environment for statistical computing Vienna, Austria. Retrieved from https://www.r-project. org/. 2017.

28 Oksanen J, Blanchet FG, Friendly M, Kindt R, Legendre P, McGlinn D, et al. Vegan: community Ecology Package. Available: https://cran.r-project.org/web/packages/vegan/index.html. Accessed: 14 April 2019.

29 Prado EL, Sebayang SK, Apriatni M, Hidayati N, Islamiyah A, Siddiq S, et al. Maternal multiple micronutrient supplementation and other biomedical and socioenvironmental influences on children's cognition at age 9-12 years in Indonesia: follow-up of the SUMMIT randomised trial. Lancet Glob Health. 2017;5:e217-28. Medline:28104188 doi:10.1016/ S2214-109X(16)30354-0

30 Chang SM, Grantham-McGregor SM, Powell CA, Vera-Hernández M, Lopez-Boo F, Baker-Henningham H, et al. Integrating a parenting intervention with routine primary health care: A cluster randomized trial. Pediatrics. 2015;136:272-80. Medline:26148947 doi:10.1542/peds.2015-0119

31 Yousafzai AK, Obradovic J, Rasheed MA, Rizvi A, Portilla XA, Tirado-Strayer N, et al. Effects of responsive stimulation and nutrition interventions on children's development and growth at age 4 years in a disadvantaged population in Pakistan: a longitudinal follow-up of a cluster-randomised factorial effectiveness trial. Lancet Glob Health. 2016;4:e548-58. Medline:27342433 doi:10.1016/\$2214-109X(16)30100-0

32 Bentenuto A, De Falco S, Venuti P. Mother-child play: A comparison of autism spectrum disorder, Down syndrome, and typical development. Front Psychol. 2016;7:1829. Medline:27920745 doi:10.3389/fpsyg.2016.01829

33 UBOS. Uganda Demographic and Health Survey. (2011). Kampala, Uganda:UBOS and Calverton (pp. 2012). Maryland: ICF International Inc.

34 Carlson AL, Xia K, Azcarate-Peril MA, Goldman BD, Ahn M, Styner MA, et al. Infant gut microbiome associated with cognitive development. Biol Psychiatry. 2018;83:148-59. Medline:28793975 doi:10.1016/j.biopsych.2017.06.021 
35 Blanton LV, Barratt MJ, Charbonneau MR, Ahmed T, Gordon JI. Childhood undernutrition, the gut microbiota, and microbiota-directed therapeutics. Science. 2016;352:1533. Medline:27339978 doi:10.1126/science.aad9359

36 Schroeder BO, Backhed F. Signals from the gut microbiota to distant organs in physiology and disease. Nat Med. 2016;22:1079-89. Medline:27711063 doi:10.1038/nm.4185

37 Chassaing B, Vijay-Kumar M, Gewirtz AT. How diet can impact gut microbiota to promote or endanger health. Curr Opin Gastroenterol. 2017;33:417-21. Medline:29019865 doi:10.1097/MOG.0000000000000401

38 Cong X, Xu W, Romisher R, Poveda S, Forte S, Starkweather A, et al. Gut microbiome and infant health: brain-gut-microbiota axis and host genetic factors. Yale J Biol Med. 2016;89:299-308. Medline:27698614

39 Cong X, Henderson WA, Graf J, McGrath JM. Early life experience and gut microbiome: The brain-gut-microbiota signaling aystem. Adv Neonatal Care. 2015;15:314-23. Medline:26240939 doi:10.1097/ANC.0000000000000191

40 FANTA-2. The analysis of the nutrition situation in Uganda. Food and nutrition technical assistance II Project. Washington, DC: AED; 2010.

41 Wamani H, Nordrehaug A, Strøm A, Peterson S, James T, Tylleskar T. Predictors of poor anthropometric status among children under 2 years of age in rural Uganda. Public Health Nutr. 2006;9:320. Medline:16684383 doi:10.1079/ PHN2006854

42 Kikafunda JK, Agaba E, Bambona A. Malnutrition amidst plenty: an assessment of factors responsible for persistent high levels of childhood stunting in food secure Western Uganda. AFJAND. 2014;14:9288313.

43 Grantham-McGregor SM, Fernald LC, Kagawa RM, Walker S. Effects of integrated child development and nutrition interventions on child development and nutritional status. Ann N Y Acad Sci. 2014;1308:11-32. Medline:24673166 doi:10.1111/nyas.12284

44 Rahman A, Fisher J, Bower P, Luchters S, Tran T, Yasamy MT, et al. Interventions for common perinatal mental disorders in women in low- and middle-income countries: a systematic review and meta-analysis. Bull World Health Organ. 2013;91:593-601I. Medline:23940407 doi:10.2471/BLT.12.109819 